\title{
Pulmonary Function, Physical Activity and Quality of Life in Chronic Obstructive Pulmonary Disease Patients: Secondary Data Analysis, Data from the Korea National Health and Nutrition Examination Survey 2015-2018
}

Kyeongbong Lee

Kangwon National University

GyuChang Lee ( $\square$ leegc76@hanmail.net)

Kyungnam University

Research Article

Keywords: COPD, Pulmonary Function, Physical Activity, Quality of Life, Secondary Data Analysis

Posted Date: December 8th, 2021

DOI: https://doi.org/10.21203/rs.3.rs-1069651/v1

License: (c) (1) This work is licensed under a Creative Commons Attribution 4.0 International License.

Read Full License 


\section{Abstract \\ Background}

COPD Patients may experience reduced physical activity and quality of life due to decreased pulmonary function. The purpose of this study is to analysis the level of pulmonary function, physical activity, and quality of life of COPD in South Korea.

\section{Methods}

This study collected the published data of the Korea National Health and Nutrition Examination Survey in 2015-2018. Out of a total of 31,648 COPD patients, the data of 121 patients were analyzed. For the pulmonary function, the data of forced vital capacity (FVC), forced expiratory volume in 1 second (FEV1), FEV6, and peak expiratory flow were collected. Physical activity was identified as frequency and duration of high/medium intensity, walking days, and strengthening exercise per week. For the quality of life (QoL), EQ-5D-3L were collected, and the frequency and index of the Korean version were collected.

\section{Results}

In the health-related characteristics, the prevalence of hypertension and asthma in COPD was relatively higher compared to other diseases. In pulmonary function, the predicted FEV1 and FEV1/FVC ratios were lower than age and weighted matched normal values. COPD showed to perform very low levels of high/medium physical activity and sitting time was confirmed to be more than 8 hours a day. In QoL, it was found that the highest reporting rate of some problems was the "pain and discomfort".

\section{Conclusion}

It was confirmed that COPD showed lower predicted FEV1 and FEV1/FVC ratios than age and weightmatched normal pulmonary function. Also, they had lowered physical activities and longer sitting time per day.

\section{Background}

Chronic obstructive pulmonary disease (COPD) is characterized by chronic airflow limitation and airway inflammation that is not fully reversible and is progressive [1]. COPD is occurring worldwide, and it is a disease that has a large national burden on the health-care system. However, studies on the specific features of COPD patients are insufficient, it is needed to investigate other health-related characteristics.

Poorly reversible expiratory airflow limitation on spirometry is an important feature of COPD [2]. The assessment of pulmonary function is the most important index of respiratory impairment in COPD [3], the 
severity of COPD can be measured by the results of spirometry airflow or pulmonary function test (PFT). Most of the PFTs which are recently practiced clinically are a method that can measure the pulmonary function of COPD patients [4]. To date, there is no specific test for physiologic perspective as COPD other than PFT, the PFT using a spirometer is a useful tool for assessing the pulmonary function.

The COPD is correlated with other factors as well as pulmonary function. Physical activity refers to the voluntary movement of the musculoskeletal system necessary for daily life [5], it can be assessed by the quantification of these voluntary movements during the way of life. The COPD patients have decreased physical activity early in the disease as well as lowered spirometry-based PFTs values [6]. Physical

activity includes basic, everyday tasks required for personal self-care and independent daily living [7], they have meaningful lower levels of physical activity compared with healthy individuals [8]. In COPD, quality of life (QoL) is an important factor of disease management, function status, and well-being, which is a subjective awareness related to the physical and mental aspects of daily life [9]. Thus, as the severity of COPD increases, the patient's physical activities and quality of life can decrease in sequence.

Therefore, the purpose of this study is to investigate the pulmonary function, physical activity, and QoL of COPD patients to identify the features of COPD patients to use them as basic data for COPD patients by survey the specific features and health-related characteristics.

\section{Materials And Methods}

\section{Data collection}

This study was based on data from the Korea National Health and Nutrition Examination Survey (KNHANES) in 2015-2018. The KNHANES is a cross-sectional and national survey accompanied by the Division of Chronic Disease Surveillance of the Korea Center for Disease Control and Prevention in the Ministry of Health and Welfare. We collected all of the 4 years of data, 31648 COPD patients in the 24hour recall method of nutrition, health, and survey. We excluded the data from participants without COPD, the data from 121 COPD patients were selected in the present study (Figure 1). This study used the data of KNHANES, which was conducted after the approval of the institutional review board in Korea Centers for Disease Control and Prevention (2018-01-03-P-A). Also, it is represented as statistical data in South Korea, which is extracted every year by stratified, clustered, and systematic sampling.

\section{Anthropometric and health-related characteristics}

Information for the anthropometry (Table 1) and health-related characteristics (Table 2) was attained through the health interview in the KNHANES. The anthropometry had age, height, weight, waist circumference, and body mass index. The subjective health status was asked by using questionnaire and the participants selected from very good, good, normal, bad, very bad, and don't know for the "How do you usually feel about your health?". The health-related characteristics surveyed the presence and absence of 
high blood pressure, diagnosis of depression, hypertension, lipidemia, stroke, myocardial infarction, asthma, and diabetes mellitus through questionnaire.

\section{Pulmonary function}

PFT is a non-invasive diagnostic test, it provides information on the quality of lung function. This test provides lung volume, capacities, and flow rate, etc. PFTs were completed as recommendation of the American Thoracic Society [10]. Dry rolling seal Spirometer (Ohio No. 822; Ohio Medical Products; Madison, Wis) was used for PFTs of participants in 2015. From 2016 to 2018, Vyntus Spirometer (Master Screen PFT, Hochberg, Germany) was used. The evaluated values of this study are forced vital capacity (FVC), predicted FVC ratio, forced expiratory volume in 1 second (FEV1), predicted FEV1 ratio, percentage of FEV1/FVC, forced expiratory volume in 6 seconds (FEV6), forced expiratory flow (FEF) $25-75 \%$ and peak expiratory flow (PEF). Spirometry values were presented as a percentage of the measured to the predicted ratio (Table 3$)$.

\section{Physical Activity}

For physical activity, items related to work, place movement, and leisure were investigated. Types of physical activity include walking days, continuous walking time at once, strengthening exercise, and sedentary time per day [8]. In the present study, high/medium intensity of physical activity, daily walking, continuous walking time at once, strengthening exercise for sports, exercise, and leisure of COPD patients were inquired.

The question of high/medium intensity of physical activity is "Do you usually do high/medium-intensity sports, exercise, and recreational activities that keep you short of breath or your heart beats very fast for at least 10 minutes?" Further, a 7-point scale (i.e., $1=$ Never, $2=1$ day, $3=2$ days, $4=3$ days, $5=4$ days, 6 $=5$ days, $7=6$ days, $8=$ everyday) was added to for walking question. These raw data were categorized as " 1 = Never and 2 = Participation in walking" for this study. Only the subjects who answered "Yes" for the time of high/medium intensity of physical activity were averaged. The daily walking time and strengthening exercise were investigated in the same way. Continuous walking time at once and sitting time were averaged of the subjects who responded to the question. Also, activity restriction due to respiration problems and smoking status was examined.

\section{Quality of life}

Investigation of QoL was performed using the Korean version of the self-administered EQ-5D-3L questionnaire. EQ-5D-3L is a generic questionnaire used to assess health-related QoL. It is comprised of a descriptive system (utility score) which asks the respondent to consider and rate his/her health 'today'. The descriptive system enables the respondent to classify his/her health consistent with five dimensions: 
mobility, self-care, usual activities, pain/discomfort, and anxiety/depression with 3 levels as follows: 1, no problems; 2, some or moderate problems; and 3, extreme problems [11]. The patient's individual health status is expressed through one answer in each of the five dimensions of EQ-5D-3L, and a total of 243 health conditions can be expressed. Also, these health statuses are converted into an EQ-5D-3L index that is calculated using the estimated weighted quality value for Koreans. The index scores range from -0.171 mean severe problems were reported for all five EQ-5D health domains, to 1 that is no problems were reported for all five EQ-5D domains. The negative scores means worse than death, 0 represents death, and 1 indicates perfect health. The validity and reliability of the Korean version of the EQ-5D-3L have been recognized across varied diseases $[12,13]$.

\section{Statistical analysis}

The data of the present study were analyzed with consideration for stratification, sampling weights, and clustering according to the KNHANES data analysis guidelines. Before statistical analysis, the Kolmogorov-Smirnov test was performed to assess the normality of continuous data. Descriptive information was obtained to examine the general characteristics of COPD patients and presented as frequencies and weighted percentages for categorical variables, and continuous variables were presented as estimated mean \pm standard errors. In the EQ-5D-3L, the categorical variables were presented as unweighted numbers and estimated percentages. Index of EQ-5D-3L was calculated as Korean version. Statistical analysis was performed using SPSS version 23.0 (IBM Corp., Armonk, NY, USA).

\section{Results}

Table 1. demonstrates the anthropometry and subjective health status of COPD patients. Of the 121 subjects, 5 were very good, 12 were good, 45 were normal, 33 were bad, 24 were very bad, and 2 were nonresponse.

Health-related characteristics and smoking status are revealed in Table 2. Diagnosis rates of hypertension (37.2\%) and asthma (33.9\%) were higher than those of other diseases. Also, the current smoking rate was $14.0 \%$ and the smoking cessation rate was $47.1 \%$.

Table 3. demonstrates the pulmonary function of the participants. FVC was $3.172 \pm 0.965 \ell$, predicted FVC was $82.07 \pm 16.71 \%$, FEV1 was $2.086 \pm 0.821 \ell$, and predicted FVC was $72.63 \pm 22.29 \%$. The FEV1/FVC ratio was $65.35 \pm 14.85 \%$, FEV6 was $2.944 \pm 0.930 \ell$, FEF $25-75 \%$ was $1.521 \pm 1.098 \ell$, and PEF was $5.404 \pm 2.279$ $\ell$.

The features of physical activity in COPD patients were described in Table 4. The subjects who performed high/medium intensity of physical activity were $8.3 / 18.2 \%$. Subjects performing high intensity physical activity did 3.3 days a week and an average of 43.5 minutes. In subjects performing medium intensity of physical activity, 3.82 days per week and an average of 38.41 minutes were completed. The frequency of 
daily walking was 2.54 days per week, continuous-time at once was 55.34 minutes. The strengthening exercise was achieved 0.93 days a week, and sitting time was 498.89 minutes a day.

Table 5. reveals the QoL by using EQ-5D-3L of the COPD patients. In five dimensions of EQ-5D-3L: mobility, self-care, usual activities, pain/discomfort, and anxiety/depression. $38.1 \%$ and $31.3 \%$ of some problems were reported in the "pain and discomfort" and "mobility". In "usual activities" showed some problem of $22.9 \%$, "anxiety and depression" had $17.8 \%$, and "self-care" was $10.1 \%$ of COPD patients demonstrated some problems.

\section{Discussion}

The present study investigated the data of the pulmonary function, physical activity, and QoL that of 121 COPD patients from the 31,649 test results of the KNHANES in 2015-2018. In subjective health status, those who answered "very good" to "normal" were $51.2 \%$, and the subjects who answered "bad" and "very bad" were $47.0 \%$. There was no specific feature because the proportion of good and bad in the selfassessed health status of COPD patients was almost similar. In physical activity that can represent physical health, only less than $20 \%$ of COPD patients performed regular physical activity. In addition, it was confirmed that the subjects spent more than 8 hours per day sitting on average. The Korean COPD patients had greater difficulty in "pain and discomfort" and "mobility".

In the health-related characteristics examined in this study, more than $30 \%$ of the COPD subjects had hypertension and asthma. Pulmonary function impairment was found to be associated with a more comorbid disease, Global Initiative for Obstructive Lung Disease stage 3 or 4 COPD had a higher prevalence of diabetes, hypertension, and cardiovascular disease [14]. In the health-related characteristics investigated in this study, COPD patients with a history of myocardial infarction or angina pectoris and diabetes were $10.7 \%$ and $13.2 \%$, whereas those with hypertension were found to be relatively high as $37.2 \%$. This study showed similar results that the proportion of COPD patients with hypertension was relatively high compared to other diseases, such as COPD patients of the United States [14].

To assess the crucial functioning of the lungs, PFT is essential. The American College of Physicians, American College of Chest Physicians, American Thoracic Society, and European Respiratory Society had recommended that spirometry can be used to identify airflow obstruction in patients with respiratory symptoms [15]. The present study also evaluated pulmonary function using a spirometer to assess airway obstruction. Since there is no data on the normal value of Asians, it was compared with the data of the most similar Caucasians to compare the results of this study by setting the values corresponding to the average height and weight of all subjects as normal values. The normal value of FVC was 3.39 $\ell[16]$, and the predicted FVC was $80-120 \%$ [17]. The results of present study demonstrated that the average of FVC was $3.17 \ell$, which was $0.22 \ell$ lower than the normal value. Predicted FVC was found to be in the lowest normal range with $82.07 \%$. The normal FEV1 value is 2.54 [16], and the predicted FEV1 is 80 $120 \%$ [17]. The FEV1 of Korean COPD patients was $0.59 \mathrm{l} / \mathrm{s}$ low, and the predicted FEV1 was $72.63 \%$, which was $7.37 \%$ lower than the lowest normal value. The normal value of the FEV1/FVC ratio was 
$75.8 \%$, and the average of Korean COPD patients was $10.45 \%$ lower. The normal level of FEV6 was 3.20 $\mathrm{l} / \mathrm{s}$, which was $0.26 \mathrm{l} / \mathrm{s}$ low in Korean COPD patients, and $2.15 \mathrm{l} / \mathrm{s}$ in FEF 25-75\%, while $1.52 \mathrm{l} / \mathrm{s}$ in Korean COPD patients was $0.63 \mathrm{l} / \mathrm{s}$ low. Lastly, the normal level of PEF was $6.72 \mathrm{l} / \mathrm{s}$, but the Korean COPD patient was $5.40 \mathrm{l} / \mathrm{s}$, which was $1.32 \mathrm{l} / \mathrm{s}$ low. As a result of this study, the average of all pulmonary functions except predicted FEV1 was lower than the normal value. It was possible to confirm the low pulmonary function value of Korean COPD patients since the FEV1/FVC ratio was $10.45 \%$ lower than normal. Comparing the average value of Korean COPD patients with the normal range, it can be considered that FEV1 is an important variable for confirming airway obstruction.

Physical activity can be defined as the amount of energy expenditure by the movement of skeletal muscles [18]. It is dependent on various factors such as physiologic, behavioral, social, environmental, and cultural aspects. Besides, physical activities are correlated with maximal walked distance, level airway obstruction, and physical health status, the present study surveyed the physical activity of COPD patients.

Physical activity is characterized by type, intensity, duration, pattern, routine, and activity-related symptoms [19]. COPD patients have significantly lowered physical activity than healthy subjects [8]. To survey the level of physical activity of COPD patients, we examined high/medium intensity of physical activity related to sports, exercise, and leisure activities. Also, daily walking, continuous walking time, strengthening exercise, and sitting time a day were observed.

Only $8.3 \%$ of the subjects performed high intensity of physical activity and $18.2 \%$ of medium-intensity. Daily walking was 3.54 days per week, and continuous walking time at once was 55.34 minutes. In particular, the strengthening exercise was found to be less than a day per week, and the average sitting time per day was 8 hours and 18 minutes. Although only 36 of the subjects responded to the survey, it was confirmed that about half of the COPD patients who responded to the questionnaire had daily activity restrictions due to respiratory problems. We thought that it is important to improve the pulmonary function of COPD patients because restriction of daily activity is associated with lowering of physical activity [6].

In previous studies, physical activity levels can predict important outcomes in COPD, lower physical activity levels are associated with a higher risk of an exacerbation-related hospitalization [20,21]. As a result, patients with COPD due to lowered levels of physical activity increase the risk of all-cause mortality after controlling for relevant confounding factors such as admission and exacerbation [22]. From the results of this study, it is considered that COPD patients need more regular and diverse physical activities and decrease sitting time a day.

Due to the characteristics of COPD, such as airway obstruction, the patients gradually decrease daily activities. Thus, health-related QoL tends to decrease with COPD severity worsen [23], and impaired health status is a risk factor for exacerbations of the respiratory problem and hospitalization [24]. The present study surveyed the EQ-5D-3L data of KNHANES, the highest percentage of reporting some problems was "pain and discomfort". The EQ-5D-3L scores are correlated with validated measures of disease severity 
including COPD admission, comorbidities, and physical activity [25]. The health-related QoL may also change depending on variations of pulmonary function and physical activity in COPD patients [22, 26], it can be an important factor to manage the QoL of COPD patients by improving the level of pulmonary function and physical activity. The present study showed that over $30 \%$ complained of some problems in the "pain and discomfort" and "mobility" category of EQ-5D-3L. Considering that the rate of complaining of discomfort to "mobility" was high, it is thought the improvement of the quality of life is necessary by increasing the activity of COPD patients such as exercise and pulmonary rehabilitation. In future studies, it is considered necessary to compare the QoL with subjects without COPD.

In Korean COPD patients, the FEF1/FVC ratio was found to be lower than the normal range (>80\%), and the degree of physical activity was also confirmed to be low. Therefore, it is considered that Korean COPD patients need medical treatment to improve pulmonary function and increase the frequency of physical activity for their improving the QoL.

\section{Limitations of this study}

This study has several limitations. First, the results of the present study may not be generalized to patients with COPD in other countries because many studies presented were related to South Korea. Another limitation, since the data of physical activity and QoL of KNHANES is a cross-sectional and selfreporting questionnaire, the results of this study is having weak evidence for COPD patients. Therefore, longitudinal studies are needed to confirm the exact level of physical activities and QoL. The third limitation was that this study compared the physical activities and health-related QoL with COPD patients, other factors had to be investigated to clarify the specific features of COPD. However, these factors affect COPD patients' medical status, daily life, future research may be necessary to determine the specific features that affect COPD patients or depending on the patient's condition.

\section{Conclusion}

Hypertension and asthma were high in the health characteristics of Korean COPD patients, and half of the respondents complained of activity restriction due to respiratory problems. Pulmonary function was found to have lower predicted FEV1 and FEV1/FVC ratios than normal values, high/medium intensity of

physical activity was very low, and sitting time a day was more than 8 hours. In QoL, it was found that the highest reporting rate of some problems was the "pain and discomfort". Since Korean COPD patients have low levels of pulmonary function and physical activity, medical treatment for this and the degree of physical activity should be improved.

\section{Declarations}

\section{Ethics approval and consent to participate}

All procedures performed in studies involving human participants were in accordance with the ethical standards of Kyungnam University Institutional Review Board and with the 1964 Helsinki declaration and 
its later amendments or comparable ethical standards.

\section{Consent for Publication}

Not applicable.

\section{Availability of data and material}

The datasets used and/or analysed during the current study are available from the corresponding author on reasonable request.

\section{Competing interests}

The authors have no conflict of interest.

\section{Funding}

This work was supported by the National Research Foundation of Korea (NRF) grant funded by the Korea government (2020R1G1A1014221).

\section{Authors' contributions}

All authors were involved in the conception and/or design of the study, the acquisition and analyzation of the data, drafted the manuscript.

\section{Acknowledgements}

Not applicable.

\section{References}

1. Rabe KF, Hurd S, Anzueto A, et al. Global strategy for the diagnosis, management, and prevention of chronic obstructive pulmonary disease: GOLD executive summary. Am J Respir Crit Care Med 2007; 176(6): 532-55.

2. O’Donnell DE, Laveneziana P, Webb K, et al. Chronic obstructive pulmonary disease: clinical integrative physiology. Clin Chest Med 2014; 35(1): 51-69.

3. Eisner $\mathrm{MD}$, Iribarren $\mathrm{C}$, Yelin $\mathrm{EH}$, et al. Pulmonary function and the risk of functional limitation in chronic obstructive pulmonary disease. Am J Epidemiol 2008; 167(9): 1090-101.

4. Pellegrino R, Viegi G, Brusasco V, et al. Interpretative strategies for lung function tests. Eur Respir J 2005; 26: 948-68.

5. Steele BG, Belza B, Cain K, et al. Bodies in motion: monitoring daily activity and exercise with motion sensors in people with chronic pulmonary disease. J Rehabil Res Dev 2003; 40(5): 45-58. 
6. Van Remoortel $\mathrm{H}$, Hornikx M, Demeyer $\mathrm{H}$, et al. Daily physical activity in subjects with newly diagnosed COPD. Thorax 2013; 68(10): 962-3.

7. Howley ET. Type of activity: resistance, aerobic and leisure versus occupational physical activity. Med Sci Sports Exerc 2001; 33(6): S364-9; discussion S419.

8. Pitta F, Troosters T, Spruit MA, et al. Characteristics of physical activities in daily life in chronic obstructive pulmonary disease. Am J Respir Crit Care Med 2005; 171(9): 972-7.

9. Blakemore A, Dickens C, Guthrie E, et al. Depression and anxiety predict health-related quality of life in chronic obstructive pulmonary disease: systematic review and meta-analysis. Int J Chron Obstruct Pulmon Dis 2014; 9: 501.

10. Society AT. Standardization of spirometry, 1994 update. Am J Respir Crit Care Med 1995; 152(3): 1107-36.

11. Brooks R, Rabin R, De Charro F. The measurement and valuation of health status using EQ-5D: a European perspective: evidence from the EuroQol BIOMED Research Programme: Springer Science \& Business Media; 2013.

12. Lee EH, Kim CJ, Cho SY, et al. Monitoring the Use of Health-Related Quality of Life Measurements in Korean Studies of Patients with Diabetes. J Korean Acad Nurs 2011; 41(4): 558-67

13. Kim SH, Hwang JS, Kim TW, et al. Validity and reliability of the EQ-5D for cancer patients in Korea. Suppor Care Cancer 2012; 20: 3155-60.

14. Mannino DM, Thorn D, Swensen A, et al. Prevalence and outcomes of diabetes, hypertension and cardiovascular disease in COPD. Eur Respir J 2008; 32: 962-9.

15. Qaseem A, Wilt TJ, Weinberger SE, et al. Diagnosis and management of stable chronic obstructive pulmonary disease: a clinical practice guideline update from the American College of Physicians, American College of Chest Physicians, American Thoracic Society, and European Respiratory Society. Ann Intern Med 2011; 155(3): 179-91.

16. Townsend MC, Occupational, Committee ELD. Spirometry in the occupational health setting-2011 update. J Occup Environ Med 2011; 53(5): 569-84.

17. Barreiro T, Perillo I. An approach to interpreting spirometry. Am Fam Physician 2004; 69(5): 1107-14.

18. Caspersen CJ, Powell KE, Christenson GM. Physical activity, exercise, and physical fitness: definitions and distinctions for health-related research. Public Health Rep 1985; 100(2): 126.

19. Spina G, Casale P, Albert PS, et al. Identifying physical activity profiles in COPD patients using topic models. IEEE J Biomed Health Inform 2015; 19(5): 1567-76.

20. Garcia-Aymerich J, Lange P, Serra I, et al. Time-dependent confounding in the study of the effects of regular physical activity in chronic obstructive pulmonary disease: an application of the marginal structural model. Ann Epidemiol 2008; 18(10): 775-83.

21. Benzo RP, Chang $\mathrm{C}-\mathrm{CH}$, Farrell $\mathrm{MH}$, et al. Physical activity, health status and risk of hospitalization in patients with severe chronic obstructive pulmonary disease. Respiration 2010; 80:10-18. 
22. Waschki B, Kirsten A, Holz O, et al. Physical activity is the strongest predictor of all-cause mortality in patients with COPD: a prospective cohort study. Chest 2011; 140(2): 331-42.

23. Ståhl E, Lindberg A, Jansson S-A, et al. Health-related quality of life is related to COPD disease severity. Health Qual Life Outcomes 2005; 3(56): 1-8.

24. Doll H, Grey-Amante P, Duprat-Lomon I, et al. Quality of life in acute exacerbation of chronic bronchitis: results from a German population study. Respir Med 2002; 96(1): 39-51.

25. Esquinas C, Ramon MA, Nuñez A, et al. Correlation between disease severity factors and EQ-5D utilities in chronic obstructive pulmonary disease. Qual Life Res 2020; 30: 607-17.

26. Westwood M, Bourbeau J, Jones PW, et al. Relationship between FEV 1 change and patient-reported outcomes in randomised trials of inhaled bronchodilators for stable COPD: a systematic review. Respir Res 2011; 12(40): 1-9.

\section{Tables}

Table 1. Anthropometry of chronic obstructive pulmonary disease patients

\begin{tabular}{|ll|}
\hline Anthropometry & $75(62.0 \%) / 46(38.0 \%)$ \\
\hline Sex (male/female) & $65.46 \pm 10.76$ \\
\hline Age (years) & $162.19 \pm 8.99$ \\
\hline Weight $(\mathrm{cm})$ & $60.75 \pm 10.46$ \\
\hline Waist circumference $(\mathrm{cm})$ & $83.18 \pm 9.86$ \\
\hline BMI (kg/m $\left.{ }^{2}\right)$ & $23.04 \pm 3.12$ \\
\hline SBP & $124.26 \pm 16.08$ \\
\hline DBP & $74.26 \pm 9.76$ \\
\hline Pulse Pressure & $34.27 \pm 25.96$ \\
\hline Subjective health status & \\
\hline Very good & $5(4.1 \%)$ \\
\hline Good & $12(9.9 \%)$ \\
\hline Normal & $45(37.2 \%)$ \\
\hline Bad & $33(27.2 \%)$ \\
\hline Very bad & $24(19.8 \%)$ \\
\hline Non-response & $2(1.7 \%)$ \\
\hline
\end{tabular}


Note: Data presented as the number (weighted \%) for categorical variables or as the mean \pm standard deviation for continuous variables. Missing values for each test item were excluded.

Abbreviation: BMI, body mass index; SBP, systolic blood pressure; DBP, diastolic blood pressure.

Table 2. Health-related characteristics of chronic obstructive pulmonary disease patients

\begin{tabular}{|llll|}
\hline Health-related diseases & Yes & No & Don't know \\
\hline Depression diagnosis & $6(5.0 \%)$ & $113(93.4 \%)$ & $2(1.7 \%)$ \\
\hline HTN diagnosis & $45(37.2 \%)$ & $76(62.8 \%)$ & $0(0.0 \%)$ \\
\hline Lipidemia diagnosis & $35(28.9 \%)$ & $86(71.1 \%)$ & $0(0.0 \%)$ \\
\hline Stroke diagnosis & $5(4.1 \%)$ & $114(94.2 \%)$ & $2(1.7 \%)$ \\
\hline Ml or angina pectoris diagnosis & $13(10.7 \%)$ & $106(87.6 \%)$ & $2(1.7 \%)$ \\
\hline Asthma diagnosis & $41(33.9 \%)$ & $78(64.5 \%)$ & $2(1.7 \%)$ \\
\hline DM diagnosis & $16(13.2 \%)$ & $105(86.8 \%)$ & $0(0.0 \%)$ \\
\hline Smoking status & & & \\
\hline Never & $47(38.9 \%)$ & & \\
\hline Current smoking & $17(14.0 \%)$ & & \\
\hline Smoking cessation & $57(47.1 \%)$ & & \\
\hline
\end{tabular}

Note: Data presented as the number (weighted \%) for categorical variables. Missing values for each test item were excluded.

Abbreviation: HTN, hypertension; MI, myocardial infarction; DM, diabetes mellitus.

Table 3. Differences in pulmonary functions of chronic obstructive pulmonary disease patients 


\begin{tabular}{|ll|}
\hline Pulmonary function & \\
\hline FVC $(\ell)$ & $3.172 \pm 0.965$ \\
\hline Predicted FVC $(\%)$ & $82.07 \pm 16.71$ \\
\hline FEV1 $(\ell)$ & $2.086 \pm 0.821$ \\
\hline Predicted FEV1 $(\%)$ & $72.63 \pm 22.29$ \\
\hline FEV1/FVC $(\%)$ & $65.35 \pm 14.85$ \\
\hline FEV6 $(\ell)$ & $2.944 \pm 0.930$ \\
\hline FEF $25-75 \%(\ell / s)$ & $1.521 \pm 1.098$ \\
\hline PEF $(\ell / s)$ & $5.404 \pm 2.279$ \\
\hline
\end{tabular}

Note: Values are expressed as mean \pm standard deviation. Missing values for each test item were excluded.

Abbreviations: FVC, forced vital capacity; FEV1, forced expiratory volume in 1 second; FEV6, forced expiratory volume in 6 seconds; FEF, forced expiratory flow; PEF, peak expiratory flow; N/A, Not available.

Table 4. Physical activities of chronic obstructive pulmonary disease patients 


\begin{tabular}{|ll|}
\hline Types of physical activities & \\
\hline High intensity of physical activity $(\mathrm{Y} / \mathrm{N})$ & $3.30 \pm 1.25$ \\
\hline Days/week & $43.50 \pm 22.85$ \\
\hline Minutes/day & $22(18.2 \%) / 99(81.8 \%)$ \\
\hline Medium intensity of physical activity $(\mathrm{Y} / \mathrm{N})$ & $3.82 \pm 1.47$ \\
\hline Days/week & $38.41 \pm 16.14$ \\
\hline Minutes/day & \\
\hline Daily walking & $3.54 \pm 2.87$ \\
\hline days/week & \\
\hline Continuous walking time at once & $55.34 \pm 41.39$ \\
\hline Minutes/day & \\
\hline Strengthening exercise & $0.93 \pm 1.69$ \\
\hline Days/week & \\
\hline Sitting time & $498.89 \pm 235.69$ \\
\hline Minutes/day & $83(68.6 \%) / 36(29.8 \%) / 2(1.7 \%)$ \\
\hline Daily activity restriction (Y/N/No response) & $17(14.0 \%) / 19(15.7 \%) / 85(70.3 \%)$ \\
\hline Due to respiratory problem (Y/N/No response) & \\
\hline
\end{tabular}

Note: Data presented as the number (weighted \%) for categorical variables or as the mean \pm standard deviation for continuous variables.

Table 5. Quality of life of chronic obstructive pulmonary disease patients 


\begin{tabular}{|lllllll|}
\hline Level & Mobility & $\begin{array}{l}\text { Self- } \\
\text { care }\end{array}$ & $\begin{array}{l}\text { Usual } \\
\text { activities }\end{array}$ & $\begin{array}{l}\text { Pain and } \\
\text { discomfort }\end{array}$ & $\begin{array}{l}\text { Anxiety and } \\
\text { depression }\end{array}$ & $\begin{array}{l}\text { Index } \\
\text { (Korean) }\end{array}$ \\
\hline 1 & $\begin{array}{l}81 \\
(68.6 \%)\end{array}$ & $\begin{array}{l}106 \\
(89.8 \%)\end{array}$ & $\begin{array}{l}91 \\
(77.1 \%)\end{array}$ & $73(61.9 \%)$ & $97(82.2 \%)$ & \\
\hline 2 & $\begin{array}{l}34 \\
(28.8 \%)\end{array}$ & $\begin{array}{l}11 \\
(9.3 \%)\end{array}$ & $\begin{array}{l}27 \\
(22.9 \%)\end{array}$ & $36(30.5 \%)$ & $19(16.1 \%)$ & \\
\hline 3 & 3 & 1 & $0(0.0 \%)$ & $9(7.6 \%)$ & $2(1.7 \%)$ & \\
\hline Total & $(2.5 \%)$ & $(0.8 \%)$ & & & $118(100 \%)$ & $0.907 \pm 0.101$ \\
\hline $\begin{array}{l}\text { Percentage } \\
\text { reporting some } \\
\text { Problems }\end{array}$ & $(118$ & 118 & 118 & 118 & $100 \%$ & \\
\hline
\end{tabular}

Note: Data presented as the number (weighted \%) for categorical variables or as the mean \pm standard deviation for continuous variables.

Results are for those who responded to the questionnaire. $98.5 \%$ of respondents to EQ-5D-3L.

b'Some problems' $=$ Level $2+3$

\section{Figures}


The KNHANES participants in 2015-2018 $(\mathrm{N}=31649)$

$$
\begin{aligned}
& \text { In } 2015(\mathrm{n}=7380) \\
& \text { In } 2016(\mathrm{n}=8150) \\
& \text { In } 2017(\mathrm{n}=8127) \\
& \text { In } 2018(\mathrm{n}=7992)
\end{aligned}
$$

Not involved in COPD diagnosis

$$
(\mathrm{N}=15362)
$$

Subjects involved in COPD diagnosis

$$
(\mathrm{N}=16287)
$$

Not been diagnosed in COPD

$$
(\mathrm{N}=16166)
$$

\section{Subjects with COPD}

$$
(\mathrm{N}=121)
$$

Exclusion: missing data (Not engaged in examination) Pulmonary function $(\mathrm{n}=31)$

Quality of life $(n=3)$

Pulmonary function $(\mathrm{n}=90)$

Physical Activities $(\mathrm{n}=121)$

Quality of life $(\mathrm{n}=118)$

\section{Figure 1}

Flow chart of the study of COPD participants 